\title{
Caracterização das Propriedades Reológicas e Dinâmicas de Composições de Borracha Natural com Resíduo de EVA
}

\author{
Marcia G. Oliveira, Marcelo H. Virgolino \\ Divisão de Processamento e Caracterização de Materiais, INT, RJ
}

Ana C. 0. Gomes, Bluma G. Soares, Viviane X. Moreira

IMA, UFRJ

\begin{abstract}
Resumo: O resíduo de EVA oriundo das indústrias calçadistas é um material reticulado, que apresenta grande potencial de uso como carga em composições elastoméricas. Neste trabalho, foram avaliadas as propriedades reológicas e dinâmicas das composições de borracha natural (NR) com resíduo de EVA (EVAR), determinadas com o auxílio do analisador de processamento de borracha (RPA). As composições não-vulcanizadas tiveram a sua viscosidade aumentada pela adição de teores crescentes de EVAR. Após a vulcanização essas composições apresentaram menor elasticidade que a NR pura, como evidenciado nas maiores taxas de relaxamento do módulo e nos maiores valores de tan $\delta$. Este comportamento foi confirmado pelos resultados de tensão e alongamento na ruptura.
\end{abstract}

Palavras-chave: Resíduo de EVA, reciclagem, composições elastoméricas.

\section{Characterization of Rheological and Dynamic Properties of Natural Rubber Compositions with Waste EVA}

Abstract: The EVA waste (EVAR) is a crosslinked material, which can be used as filler in rubber compositions. In this work, rheological and dynamic properties of natural rubber (NR) compositions with EVAR were investigated before and after vulcanization with the help of a rubber processing analyzer (RPA). The filler content varied from 0 to $60 \mathrm{phr}$. The results showed that rheological properties of nonvulcanized compositions were clearly modified by the addition of EVAR, which contributed to an increase in viscosity. The modulus relaxation and the dynamic properties such as $\tan \delta$ of vulcanized compositions were also affected, by the reduction of the elasticity of the compositions. The mechanical properties corroborated the behavior revealed by RPA analysis and indicated that EVAR acted as a non-reinforcing filler.

Keywords: Waste EVA, recycling, rubber compositions.

\section{Introdução}

A adoção de novos materiais e modernos processos de fabricação de calçados aumentou significativamente tanto a produção como a produtividade do setor. Porém, fez surgir na mesma escala os resíduos industriais, inaproveitáveis economicamente, degradáveis apenas após um longo período de tempo. Dentre os resíduos gerados no setor, destacam-se os retalhos e aparas do copolímero de etileno-acetato de vinila (EVA), utilizados na fabricação de solados, palmilhas e entre-solas de calçados. O processo de obtenção desses solados produz perdas da ordem de $18 \%$ em massa. Com base neste percentual, estima-se que a quantidade deste resíduo no Brasil atinja cerca de 5500 toneladas anuais ${ }^{[1]}$.

A busca de soluções do ponto de vista ecológico e econômico para a destinação final dos resíduos de EVA (EVAR) tem sido uma constante nos meios empresariais, entidades ambientalistas e vários outros segmentos sociais. A deposição a céu aberto ou em aterros sanitários não constitui uma solução definitiva para resíduos sintéticos, uma vez que a taxa de degradação e a densidade do material são muito baixas, assim como, por razões ambientais a incineração também não é recomendada ${ }^{[2-7]}$. Em nenhum lugar onde são fabricados calçados foi encontrada solução para os resíduos industriais deste material. Nos países asiáticos a quantidade destes resíduos está crescendo assustadoramente.

O EVAR apresenta um grande potencial de uso como carga em formulações elastoméricas, visto que os solados são confeccionados adicionando-se agentes expansores e reticulantes, além de cargas inorgânicas ${ }^{[8-11]}$. As cargas podem ser classificadas, de acordo com seu desempenho no elastômero, em reforço ou enchimento. O reforço no elastômero, proveniente da carga, se reflete na melhoria das propriedades mecânicas como: resistência à tração, ao rasgamento e à abrasão, quando as propriedades são comparadas com formulação semelhante, porém sem carga ${ }^{[10]}$.

Este trabalho tem o objetivo de estudar o reaproveitamento de resíduos de EVA de forma racional, visando uma utilização mais nobre para o mesmo. Desta forma, foram preparadas 
composições de borracha natural (NR) com EVAR, com teor de carga entre 0 e $60 \mathrm{phr}$. O efeito da carga e do seu teor foi avaliado através do acompanhamento das propriedades reológicas e dinâmicas, medidas no analisador de processamento de borracha (RPA), e das propriedades mecânicas.

\section{Experimental}

\section{Materiais}

A borracha natural usada foi do tipo 1 GEB (Granulado Escuro Brasileiro) com viscosidade Mooney 94,8 ML (1+4) a $100{ }^{\circ} \mathrm{C}$. O resíduo de poli(etileno-co-acetato de vinila) (EVAR) foi fornecido por indústrias calçadistas de Caxias do Sul, com as seguintes características: teor de acetato de vinila $=18 \%$; teor de gel $=65 \%$; densidade $=0,989 \mathrm{~g} / \mathrm{cm}^{3}$; cristalinidade $=$ $10,4 \mathrm{~J} / \mathrm{g}^{[13]}$. O resíduo foi recebido na forma moída e sua distribuição granulométrica encontra-se na tabela 1 .

\section{Preparação das composições NR-EVAR}

As composições NR-EVAR foram preparadas em misturador de rolos, a $60^{\circ} \mathrm{C}$, com razão de fricção 1:1,1. A formulação utilizada foi (em phr): NR (100); óxido de zinco $(\mathrm{ZnO})(5,0)$; ácido esteárico $(1,5)$; antioxidante $(1,0)$; enxofre $(2,5)$; 2,2-ditiomercaptobenzotiazol (MBTS) $(0,8)$. O teor de EVAR incorporado variou entre 0 e $60 \mathrm{phr}$.

\section{Caracterização das composições NR-EVAR}

As propriedades reológicas e dinâmicas das composições antes e após a vulcanização, bem como as medidas dos parâmetros de vulcanização, foram feitas no analisador de processamento de borracha (RPA 2000), da marca Alpha Technologies. As condições do processo de vulcanização (tempo, temperatura, arco e freqüência de oscilação) foram mantidas constantes para as diferentes composições. As condições de análise encontram-se descritas nas Tabelas 2 e 3.

Os ensaios de dureza foram realizados segundo norma ASTM D 2240 em durômetro Shore, tipo A-2. Foram feitas 5 medidas para cada composição e os resultados foram as medianas dos valores parciais.

Os ensaios de tração foram realizados em Máquina Universal de Ensaios EMIC, modelo DL 2000, segundo a norma DIN 53504, à temperatura ambiente e velocidade de separação das garras de $200 \mathrm{~mm} / \mathrm{min}$.

Análises de microscopia eletrônica de varredura (SEM)

Tabela 1. Distribuição granulométrica do resíduo de EVA (EVAR)

\begin{tabular}{cc}
\hline $\begin{array}{c}\text { Tamanho de partícula } \\
(\boldsymbol{\mu m})\end{array}$ & $\begin{array}{c}\text { Fração em massa } \\
(\boldsymbol{\%})\end{array}$ \\
\hline $\mathrm{x}<710$ & 1,94 \\
$355<\mathrm{x}<710$ & 10,93 \\
$180<\mathrm{x}<355$ & 43,52 \\
$106<\mathrm{x}<180$ & 28,03 \\
$38<\mathrm{x}<106$ & 15,58 \\
\hline
\end{tabular}

Tabela 2. Configuração da rotina de análise do RPA para determinação das propriedades das composições NR-EVAR antes da vulcanização

\begin{tabular}{ccc}
\hline Subteste 1 & \multicolumn{2}{c}{ Condicionamento } \\
\hline Tempo & $5 \mathrm{~min}$ \\
Temperatura & $100{ }^{\circ} \mathrm{C}$ \\
& Freqüência & $30 \mathrm{cpm}$ \\
& Deformação & $0,2^{\circ}$ \\
\hline Subteste 2 & Variação da Freqüiência \\
\hline & Temperatura & $100{ }^{\circ} \mathrm{C}$ \\
& Deformação & $0,5^{\circ}$ \\
& Faixa de freqüência & $2-2000 \mathrm{cpm}$ \\
\hline
\end{tabular}

Tabela 3. Configuração da rotina de análise do RPA para determinação dos parâmetros de vulcanização e das propriedades das composições NR-EVAR após a vulcanização

\begin{tabular}{|c|c|c|}
\hline \multirow[t]{5}{*}{ Subteste 1} & \multicolumn{2}{|l|}{ Cura } \\
\hline & Tempo & $12 \min$ \\
\hline & Temperatura & $160{ }^{\circ} \mathrm{C}$ \\
\hline & Freqüência & $100 \mathrm{cpm}$ \\
\hline & Deformação & $0,5^{\circ}$ \\
\hline \multirow[t]{4}{*}{ Subteste 2} & \multicolumn{2}{|c|}{ Relaxamento de tensão } \\
\hline & Tempo & $1000 \mathrm{~s}$ \\
\hline & Temperatura & $100{ }^{\circ} \mathrm{C}$ \\
\hline & Deformação & $0,75^{\circ}$ \\
\hline \multirow[t]{4}{*}{ Subteste 3} & \multicolumn{2}{|c|}{ Variação de freqüiência } \\
\hline & Temperatura & $60{ }^{\circ} \mathrm{C}$ \\
\hline & Deformação & $0,5^{\circ}$ \\
\hline & Faixa de freqüência & $2-500 \mathrm{cpm}$ \\
\hline \multirow[t]{5}{*}{ Subteste 4} & \multicolumn{2}{|c|}{ Envelhecimento } \\
\hline & Tempo & $30 \mathrm{~min}$ \\
\hline & Temperatura & $180^{\circ} \mathrm{C}$ \\
\hline & Freqüência & $200 \mathrm{cpm}$ \\
\hline & Deformação & $0,5^{\circ}$ \\
\hline \multirow[t]{4}{*}{ Subteste 5} & \multicolumn{2}{|c|}{ Variação de freqüiência } \\
\hline & Temperatura & $60{ }^{\circ} \mathrm{C}$ \\
\hline & Deformação & $0,5^{\circ}$ \\
\hline & Faixa de freqüência & $2-500 \mathrm{cpm}$ \\
\hline
\end{tabular}


foram conduzidas em microscópio JEOL 5610 LV utilizando-se detector de elétrons retroespalhados e voltagem de $10 \mathrm{kV}$. As amostras foram criogenicamente fraturadas, sendo a superfície tratada com tetróxido de ósmio $\left(\mathrm{OsO}_{4}\right)$ durante 5 min, a fim de tingir seletivamente a fase insaturada. Após o tratamento, a superfície foi coberta com uma fina camada de carbono e submetida à análise no microscópio.

\section{Resultados e Discussão}

\section{Propriedades reológicas das composições não- vulcanizadas}

$\mathrm{O}$ analisador de processamento de borracha (RPA) permite medir as características viscoelásticas de elastômeros crus, compostos vulcanizados e não-vulcanizados, determinando assim, o comportamento reológico dos mesmos. As propriedades medidas no RPA são as seguintes: torque elástico (S'), viscoso ( $\left.\mathrm{S}^{\prime \prime}\right)$ e complexo ( $\left.\mathrm{S}^{*}\right)$; módulo elástico (G'), viscoso $\left(G^{\prime \prime}\right)$ e complexo $\left(G^{*}\right)$; tangente de perda $(\tan \delta)$; viscosidade dinâmica complexa $\left(\mathrm{n}^{*}\right)$ e real $\left(\mathrm{n}^{\prime}\right)^{[12]}$.

A Figura 1 ilustra o comportamento da viscosidade dinâmica real (n') de acordo com a variação da freqüência para composições antes da vulcanização. As quatro composições apresentam comportamento pseudoplástico típico, com a viscosidade decrescendo de acordo com o aumento da freqüência aplicada. É claramente observada a influência do EVAR pelo grande aumento da viscosidade das composições. Além disso, a tangente de perda ( $\tan \delta$ ) diminui à medida que aumenta a quantidade de EVAR nas composições, assinalando uma restrição da mobilidade das cadeias de NR, conforme pode ser visto na Figura 2. Este comportamento já era espe-

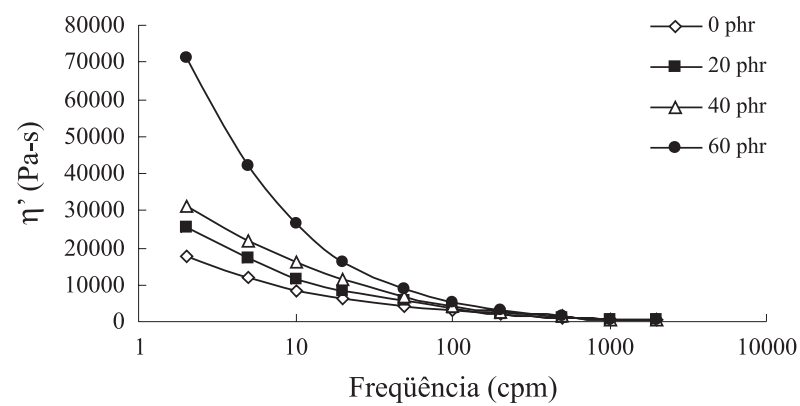

Figura 1. Variação da viscosidade real dinâmica (n') de acordo com a freqüência para as composições de NR-EVAR antes da vulcanização, medida à $100{ }^{\circ} \mathrm{C}$ com arco de oscilação de $0,5^{\circ}$.

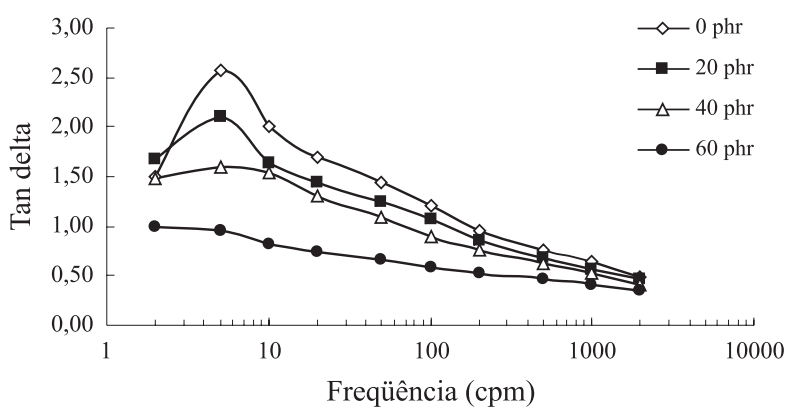

Figura 2: Variação da $\tan \delta$ em função da freqüência para as composições nãovulcanizadas de NR-EVAR, medida à $100^{\circ} \mathrm{C}$ com arco de oscilação de $0,5^{\circ}$.

rado em decorrência da natureza reticulada do resíduo e da presença de cargas inorgânicas no mesmo.

\section{Parâmetros de vulcanização}

A Tabela 4 reúne os principais parâmetros de vulcanização das composições NR-EVAR. A adição de EVAR aumenta sensivelmente os valores de torque elástico mínimo (S'mínimo). É comum associar estes valores à viscosidade das composições e sendo assim, há uma confirmação do aumento da viscosidade em função da incorporação de teores crescentes do resíduo de EVA, como discutido anteriormente. Por outro lado, os valores de torque elástico máximo (S'máximo) diminuem de acordo com a adição de EVAR. Esta tendência pode ser atribuída à menor quantidade de borracha disponível para ser vulcanizada pelo enxofre, logo os valores de S'máximo serão menores em relação à composição pura, visto que este é função direta da quantidade de ligações cruzadas formadas durante a vulcanização.

Em relação aos tempo de pré-cura $\left(\mathrm{t}_{10}\right.$ - tempo necessário para se atingir um grau de cura de $10 \%$ ) e tempo ótimo de cura $\left(\mathrm{t}_{90}\right.$ - tempo necessário para se atingir um grau de cura de $90 \%$ ), observa-se que ambos aumentam de acordo com a adição de EVAR, assinalando um retardamento do processo de vulcanização, o qual pode ser atribuído ao efeito de diluição, visto que a quantidade de agentes de vulcanização permanece constante, porém, a massa global da formulação (borracha + EVAR) aumenta. Este mesmo comportamento foi observado em misturas de elastômeros e EVA virgem ${ }^{[13]}$.

\section{Propriedades dinâmicas das composições vulcanizadas}

Os ensaios de relaxamento de tensão são muito úteis na determinação do desempenho mecânico de materiais ao longo do tempo. O RPA possibilita a realização desse tipo de

Tabela 4. Parâmetros de vulcanização das composições NR-EVAR

\begin{tabular}{|c|c|c|c|c|c|}
\hline $\begin{array}{c}\text { Teor de EVAR } \\
\text { phr }\end{array}$ & $\begin{array}{l}\text { S'mínimo } \\
\text { dN.m }\end{array}$ & $\begin{array}{l}\text { S'máximo } \\
\text { dN.m }\end{array}$ & $\begin{array}{c}S^{\prime} \min -S^{\prime} \max \\
\text { dN.m }\end{array}$ & $\begin{array}{c}\mathbf{t}_{10} \\
\mathbf{m i n}\end{array}$ & $\underset{\mathbf{m i n}}{\mathbf{t}_{90}}$ \\
\hline 0 & 0,049 & 2,285 & 2,236 & 0,78 & 2,72 \\
\hline 20 & 0,059 & 2,040 & 1,981 & 0,95 & 3,78 \\
\hline 40 & 0,078 & 1,915 & 1,837 & 1,00 & 4,30 \\
\hline 60 & 0,137 & 1,807 & 1,670 & 1,15 & 4,42 \\
\hline
\end{tabular}


ensaio através da aplicação de uma deformação angular na amostra com o acompanhamento posterior da variação do módulo de cisalhamento $(\mathrm{G})$ ao longo do tempo das composições vulcanizadas (Figura 3). É possível observar que a taxa de relaxamento do módulo aumenta de acordo com a quantidade de EVAR incorporada à NR (Tabela 5). Este aumento pode ser atribuído à redução da elasticidade nas composições com teores crescentes de EVAR, como assinalado anteriormente pelos menores valores de torque elástico máximo (S'máximo) do subteste de cura.

As variações promovidas pela adição do EVAR na elasticidade das composições de NR também podem ser verificadas através do acompanhamento da variação da tangente de perda $(\tan \delta)$ nos ensaios de varredura de freqüência, conforme mostra a Figura 4. Fica claro que a adição de EVAR ocasiona um aumento nos valores de $\tan \delta$, ou seja, há um incremento da componente viscosa em detrimento da componente elástica. Além disso, a partir dos valores de tan $\delta$ é possível especular sobre a resiliência do material e neste sentido quanto maior a quantidade de EVAR incorporada menos resiliente se torna o material. Comportamento semelhante foi relatado para composições de borracha reforçada com negro de fumo. Segundo os autores, a tan $\delta$ após a vulcanização é função direta da densidade de ligações cruzadas ${ }^{[12,14,15]}$. Assim, quantidades crescentes de carga implicam na redução da fração elastomérica na formulação, resultando em menor teor de ligações cruzadas formadas durante a vulcanização e, portanto, a elasticidade é diminuída.

Vale ressaltar que a tendência exibida pela $\tan \delta$ está de acordo com os resultados do ensaio de relaxamento de ten-

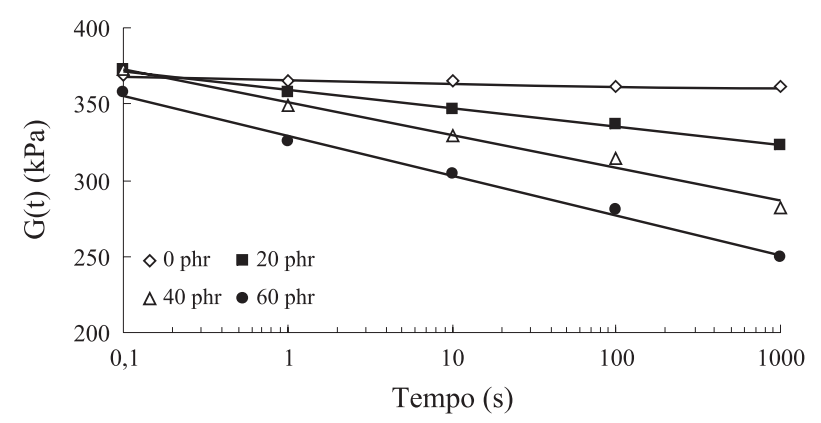

Figura 3. Curvas de relaxamento do módulo de cisalhamento (G) das composições vulcanizadas de NR-EVAR, medido a $100^{\circ} \mathrm{C}$.

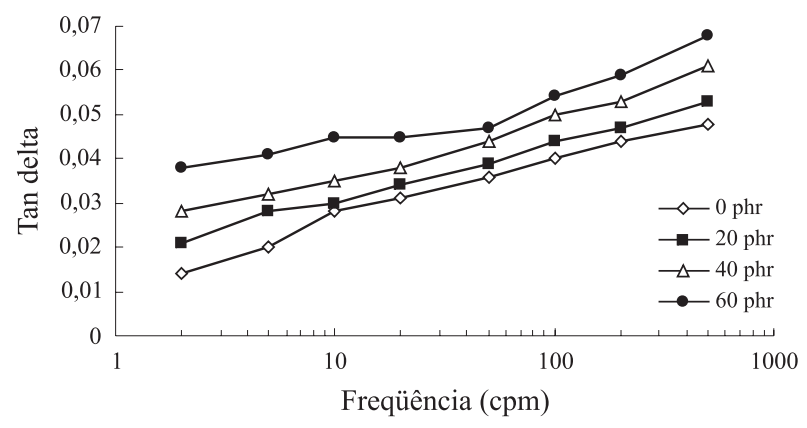

Figura 4. Variação da $\tan \delta$ em função da freqüência para as composições vulcanizadas de NR-EVAR, medida à $60^{\circ} \mathrm{C}$ e arco de oscilação de $0,5^{\circ}$.

são, no qual se observou um aumento da taxa de relaxamento em função da adição de EVAR.

\section{Envelhecimento térmico}

A Figura 5 ilustra o comportamento das composições de NR com EVAR frente à degradação térmica realizada no RPA. Para tanto, as composições foram vulcanizadas no RPA e submetidas a uma temperatura de $180^{\circ} \mathrm{C}$ durante 30 minutos, a $\tan \delta$ foi medida antes e após o processo de degradação. De acordo com os resultados obtidos, todas as composições sofrem degradação, visto que apresentam maiores valores para a tan $\delta$ após o tratamento a $180^{\circ} \mathrm{C}$. Este aumento nos valores de $\tan \delta$ indica uma maior flexibilidade do material, decorrente da quebra de cadeia induzida pela alta temperatura. Sobretudo, ressalta-se que a adição de EVAR reduz a degradação térmica da NR.

\section{Propriedades mecânicas e morfológicas}

A Tabela 6 mostra os resultados de tensão na ruptura, alongamento na ruptura e dureza das composições estudadas. Pode ser observado que os valores de dureza aumentam de acordo com a quantidade de EVAR. Esses resultados são esperados devido à própria natureza termoplástica do EVAR, associada à alta proporção de material reticulado no resíduo. A tensão na ruptura permanece inalterada até um teor de $40 \mathrm{phr}$ de EVAR, diminuindo ligeiramente para quantidades maiores de EVAR. $O$ alongamento na ruptura diminui gradativamente com o aumento do teor de resíduo. Estes resultados podem ser atribuídos à incompatibilidade entre os dois componentes, favorecida pela diferença de polaridade. Essa incompatibilidade, associada à presença de material reticulado, dificulta

Tabela 5. Taxa de relaxamento do módulo de cisalhamento (G) das composições vulcanizadas de NR-EVAR

\begin{tabular}{ccccc}
\hline & \multicolumn{4}{c}{ Taxa de relaxamento do módulo (\%) } \\
\cline { 2 - 5 } Faixa de tempo (s) & $\mathbf{0} \mathbf{p h r} *$ & $\mathbf{2 0} \mathbf{~ p h r *}$ & $\mathbf{4 0} \mathbf{~ p h r *}$ & $\mathbf{6 0}$ phr* \\
\cline { 2 - 5 } $0,1-1$ & 8,26 & 14,78 & 20,45 & 21,58 \\
$1-10$ & 8,12 & 16,69 & 24,50 & 27,13 \\
$10-100$ & 6,90 & 20,35 & 27,67 & 32,49 \\
$100-1000$ & 6,15 & 22,64 & 33,93 & 41,08 \\
\hline
\end{tabular}

${ }^{*}$ Quantidade de EVAR 


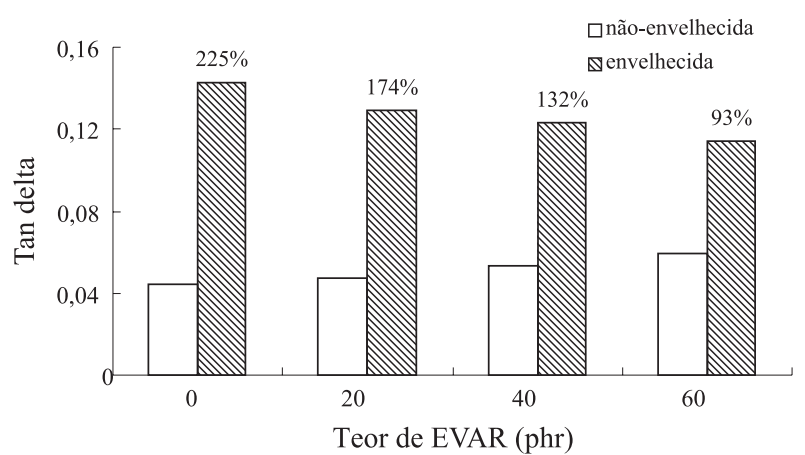

Figura 5. Variação da tangente de perda ( $\tan \delta$ ), medida a $200 \mathrm{cpm}$, das composições de NR com EVAR após degradação térmica a $180^{\circ} \mathrm{C}$.
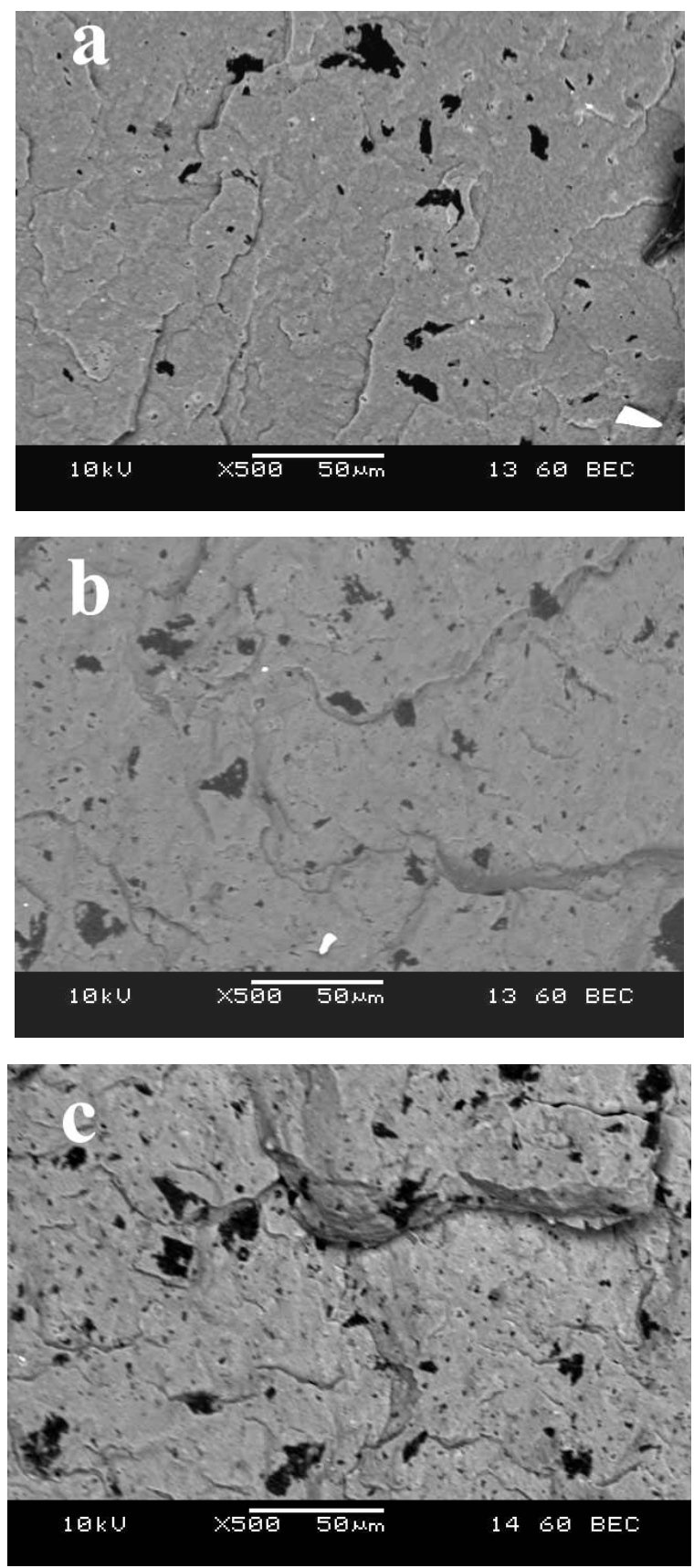

Figura 6. Microscopia eletrônica de varredura das composições vulcanizadas de NR-EVAR, contendo (a) $20 \mathrm{phr}$, (b) $40 \mathrm{phr}$ e (c) $60 \mathrm{phr}$ de EVAR
Tabela 6. Propriedades mecânicas das composições NR-EVAR

\begin{tabular}{cccc}
\hline $\begin{array}{c}\text { Teor de EVAR } \\
\text { phr }\end{array}$ & $\begin{array}{c}\text { Dureza } \\
\text { Shore A }\end{array}$ & $\begin{array}{c}\text { Tensão } \\
\text { na ruptura } \\
\text { MPa }\end{array}$ & $\begin{array}{c}\text { Alongamento } \\
\text { na ruptura } \\
\text { \% }\end{array}$ \\
\hline 0 & 34 & 19,1 & 655 \\
20 & 42 & 19,9 & 635 \\
40 & 51 & 19,4 & 615 \\
60 & 55 & 17,8 & 575 \\
\hline
\end{tabular}

a dispersão do EVAR na matriz de NR, favorecendo a formação de agregados.

O grau de dispersão de EVAR pode ser observado por microscopia eletrônica de varredura. A Figura 6 apresenta as micrografias das composições de borracha natural, contendo quantidade crescente de EVAR. A matriz de NR, tingida com $\mathrm{OsO}_{4}$, aparece clara quando se utiliza o detector de elétrons retroespalhados, enquanto que os domínios de EVAR se apresentam mais escuros. Observa-se nessas micrografias a presença de agregados de EVAR heterogeneamente distribuídos na matriz de NR. Entretanto, dentro da faixa de concentração utilizada, não foram observadas diferenças sensíveis na morfologia das misturas, o que está de acordo com os resultados encontrados de propriedades mecânicas, que também variaram pouco. Este conjunto de resultados indica que o resíduo de EVA pode ser considerado como uma carga de enchimento.

Além disso, é importante ressaltar a concordância entre as propriedades determinadas no RPA e as propriedades mecânicas convencionais, dureza e resistência à tração.

\section{Conclusões}

As propriedades reológicas das composições nãovulcanizadas foram modificados pela adição do resíduo de EVA, provavelmente em função da sua natureza reticulada e da presença de aditivos inorgânicos, que em conjunto propiciaram o aumento da viscosidade e da elasticidade dessas composições, tornando o processamento mais difícil. Além disso, também foram observadas alterações no processo de cura, que se tornou mais lento à medida que foram adicionadas à NR quantidades crescentes de EVAR.

Como esperado, as propriedades dinâmicas das composições vulcanizadas também foram afetadas pela incorporação de EVAR. Este último contribuiu para a redução da elasticidade, conforme evidenciado pelos maiores valores de taxa de relaxamento do módulo e de $\tan \delta$. Acredita-se que a redução da quantidade de borracha presente nas composições NR-EVAR seja responsável por essa redução. Esse mesmo motivo pode explicar a maior estabilidade térmica das composições contendo teores crescentes de EVAR.

Finalmente, as propriedades mecânicas traduziram bem as alterações de elasticidade reveladas nas análises de RPA, através da redução dos valores de alongamento na ruptura. Ainda com base nos ensaios de tração, chegou-se à conclusão 
que o EVAR atua como carga de enchimento na matriz de NR e sobretudo, sem grande prejuízo das propriedades mecânicas.

\section{Agradecimentos}

Os autores agradecem ao CEPG - UFRJ, CAPES, CNPq (Proc. no 130547/2000.0), PADCT (Proc. No 620132/98-1) e à FAPERJ pelo auxílio financeiro e à Universidade de Caxias do Sul pela doação do EVA reciclado.

\section{Referências Bibliográficas}

1. Vieira, C. A. B. et al. - "Caracterização de Misturas EVA/ HDPE Modificadas com Agentes Compatibilizantes", in: Anais do $5^{\circ}$ Congresso Brasileiro de Polímeros, p.305, Águas de Lindóia - SP, nov (1999).

2. Fukumori, K. et al. - JSAE Review, 23, p.259 (2002).

3. Fang, Y.; Zhan, M.; Wang, Y. - Materials and Design, 22, p.123 (2001).

4. Adhikari, B.; De, D.; Maiti, S. - Prog. Polym. Sci., 25, p.909 (2000).

5. Schnecko, H. - Macromol. Symp., 135, p.327 (1998).

6. Balogh, G.; Samay, G. - Int. Polym. Sci. Technol., 23, p.86 (1996).

7. Dierkes, W. - Intern. Polym. Sci. Technol., 22, p.17 (1995).
8. Sombatsompop, N. - J. Appl. Polym. Sci., 74, p.1129 (1999).

9. Mathew, G. et al. - J. Appl. Polym. Sci., 61, p.2035 (1996).

10. Oliphant, K.; Baker, W.E. - Polym. Eng. Sci., 33, p.166 (1993).

11. Medalia, A.I.; Kraus, G. "Reinforcement of Elastomers by Particulate Fillers" in Science and Technology of Rubber, Second Edition, cap. 8, Academic Press, New York, 1994.

12. Dick, J.S.; Harmon, C.; Vare, A. - Polym. Testing, 18, p.237 (1999).

13. Moreira, V.X., - "Utilização de Resíduo de EVA em Composições de Borracha Nitrílica", Tese de Mestrado, Universidade Federal do Rio de Janeiro (2001).

14. Dick, J.S.; Pawlowski, H.A. "Rubber Characterization by Applied Strain Variations Using the Rubber Process Analyzer", in, Proceedings of the Rubber Division, American Chemical Society, p.1, Chicago, abr (1994).

15. Dick, J.S.; Pawlowski, H.A. “Applications for the Rubber Process Analyzer", in, Proceedings of the Rubber Division, American Chemical Society, p.1, Nashville, nov (1992).

Enviado: $23 / 10 / 03$

Reenviado: $24 / 06 / 04$

Aprovado: 30/06/04 